\title{
Management of Do Not Resuscitate Orders Before Invasive Procedures
}

\author{
Jennifer Wong, MD; Amy Gravely; and Peter G. Duane, MD
}

Background: In 2017, the US Department of Veterans Affairs (VA) implemented the Life-Sustaining Treatment Decisions Initiative (LSTDI), which created a portable and durable code status for use across its health care system. Patients who now have a durable do not resuscitate (DNR) status may undergo invasive procedures. Few studies have examined whether proceduralists discuss DNR status and document changes before procedures.

Objective: To assess baseline percentage of suspension of DNR before nonsurgical invasive procedures and determine whether an academic detailing intervention consisting of training proceduralists in the use of a template that allows rapid suspension of DNR status increases percentage of DNR acknowledgments.

Methods: Single-center, quasi-experimental pre- and postassessments were done in high-volume, procedural areas, including gastroenterology, cardiology, and interventional radiology, in a VA medical center. The primary outcome was the proceduralists' documentation of DNR status acknowledgment before a nonsurgical invasive procedure at baseline and after the intervention. Logistic regression was used to compare percentage of DNR acknowledgment with time (before, after) and procedural area and assessing their interaction in the model.

Results: The interaction between department and time revealed wide variation in documentation of DNR acknowledgment. Examining the model predicted percentages from the interaction, preintervention percentages for gastroenterology, cardiology and interventional radiology were 46\%, 75.6\%, and $7.5 \%$, respectively, and postintervention model predicted percentages were $53.5 \%, 91.7 \%$, and $26.3 \%$, respectively. Only the before vs after contrast for interventional radiology was significantly different. When all procedural areas were combined, the percentage of DNR acknowledgment significantly improved from $38.6 \%$ to $61.1 \%(P=.01)$.

Conclusions: Before nonsurgical invasive procedures, the percentage of DNR acknowledgment was low but after, the intervention significantly improved. Further research is needed to assess its impact on patient-centered outcomes.

\begin{abstract}
Jennifer Wong is an Instructor, and Peter Duane is an Associate Professor, both at the University of Minnesota in Minneapolis. Amy Gravely is a Research Service Biostatistician, and Peter Duane is an Associate Director of the Primary and Specialty Care Service Line in the Division of Pulmonary and Critical Care, both at the Minneapolis Veterans Affairs Health Care System.

Correspondence: Jennifer Wong (wongx601@umn.edu)
\end{abstract}

Fed Pract. 2021;38(2):80-83. doi:10.12788/fp.0088 r n January 2017, the US Department of Veterans Affairs (VA), led by the National Center of Ethics in Health Care, created the Life-Sustaining Treatment Decisions Initiative (LSTDI). The VA gradually implemented the LSTDI in its facilities nationwide. In a format similar to the standardized form of portable medical orders, provider orders for life-sustaining treatments (POLST), the initiative promotes discussions with veterans and encourages but does not require health care professionals (HCPs) to complete a template for documentation (life-sustaining treatment [LST] note) of a patient's preferences. ${ }^{1}$ The HCP enters a code status into the electronic health record (EHR), creating a portable and durable note and order.

With a new durable code status, the HCPs performing these procedures (eg, colonoscopies, coronary catheterization, or percutaneous biopsies) need to acknowledge and can potentially rescind a do not resuscitate (DNR) order. Although the risk of cardiac arrest or intubation is low, all invasive procedures carry these risks to some degree. ${ }^{2,3}$ Some HCPs advocate the automatic discontinuation of DNR orders before any procedure, but multiple professional societies recommend that patients be included in these discussions to honor their wishes. ${ }^{4-7} \mathrm{Al}-$ though no procedures at the VA require the suspension of a DNR status, it is important to establish which life-sustaining measures are acceptable to patients.

As part of the informed consent process, proceduralists (HCPs who perform a procedure) should discuss the option of temporary suspension of DNR in the periprocedural period and document the outcome of this discussion (eg, rescinded DNR, acknowledgment of continued DNR status). These discussions need to be documented clearly to ensure accurate communication with other HCPs, particularly those caring for the patient postprocedure. Without the documentation, the risk that the patient's wishes will not be honored is high. ${ }^{8}$ Code status is usually addressed before intubation of general anesthesia; however, nonsurgical procedures have a lower likelihood of DNR acknowledgment.

This study aimed to examine and improve the rate of acknowledgment of DNR status before nonsurgical procedures. We hypothesized that the rate of DNR acknowledgment before nonsurgical invasive procedures is low; and the rate can be raised with an intervention designed to educate proceduralists and improve and simplify this documentation. ${ }^{9}$ 


\section{METHODS}

This was a single center, before/after quasiexperimental study. The study was considered clinical operations and institutional review board approval was unnecessary.

A retrospective chart review was performed of patients who underwent an inpatient or outpatient, nonsurgical invasive procedure at the Minneapolis VA Medical Center in Minnesota. The preintervention period was defined as the first 6 months after implementation of the LSTDI between May 8, 2018 and October 31, 2018. The intervention was presented in December 2018 and January 2019. The postintervention period was from February 1, 2019 to April 30, 2019.

Patients who underwent a nonsurgical invasive procedure were reviewed in 3 procedural areas. These areas were chosen based on high patient volumes and the need for rapid patient turnover, including gastroenterology, cardiology, and interventional radiology. An invasive procedure was defined as any procedure requiring patient consent. Those patients who had a completed LST note and who had a DNR order were recorded.

The intervention was composed of 2 elements: (1) an addendum to the LST note, which temporarily suspended resuscitation orders (Figure). We developed the addendum based on templates and orders in use before LSTDI implementation. Physicians from the procedural areas reviewed the addendum and provided feedback and the facility chief-of-staff provided approval. Part 2 was an educational presentation to proceduralists in each procedural area. The presentation included a brief introduction to the LSTDI, where to find a life-sustaining treatment note, code status, the importance of addressing code status, and a description of the addendum. The proceduralists were advised to use the addendum only after discussion with the patient and obtaining verbal consent for DNR suspension. If the patient elected to remain DNR, proceduralists were encouraged to document the conversation acknowledging the DNR.

\section{Outcomes}

The primary outcome of the study was proceduralist acknowledgment of DNR status before nonsurgical invasive procedures.

\section{FIGURE Life-Sustaining Treatment Template}

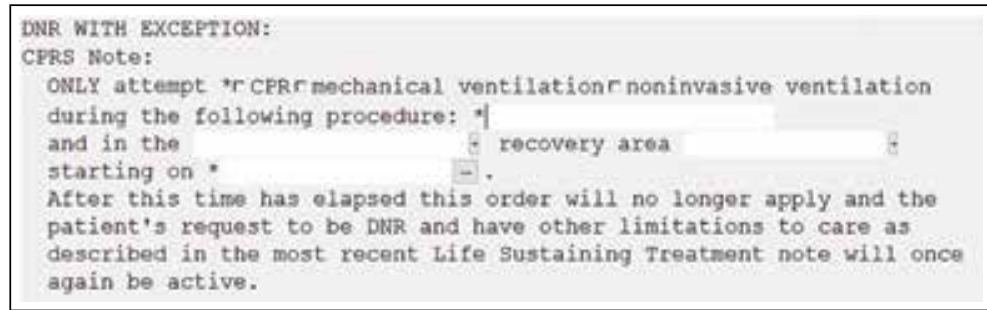

Abbreviations: CPR, cardiopulmonary resuscitation; CPRS, Computerized Patient Record System; DNR, do not resuscitate.

DNR status was considered acknowledged if the proceduralist provided any type of documentation.

\section{Statistical Analysis}

Model predicted percentages of DNR acknowledgment are reported from a logistic regression model with both procedural area, time (before vs after) and the interaction between these 2 variables in the model. The simple main effects comparing before vs after within the procedural area based on post hoc contrasts of the interaction term also are shown.

\section{RESULTS}

During the first 6 months following LSTDI implementation (the preintervention phase), 5,362 invasive procedures were performed in gastroenterology, interventional radiology, and cardiology. A total of 211 procedures were performed on patients who had a prior LST note indicating DNR. Of those, 68 (32.2\%) had documentation acknowledging their DNR status. The educational presentation was given to each of the 3 departments with about $75 \%$ faculty attendance in each department. After the intervention, 1,932 invasive procedures were performed, identifying 143 LST notes with a DNR status. Sixty-five (45.5\%) had documentation of a discussion regarding their DNR status.

The interaction between procedural areas and time (before, after) was examined. Of the 3 procedural areas, only interventional radiology had significant differences before vs after, $7.5 \%$ vs $26.3 \%$, respectively $(P=.01)$. Model-adjusted percentages before vs after for cardiology were $75.6 \%$ vs $91.7 \%(P=.12)$ and for gastroenterology were $46 \%$ vs $53.5 \%$ $(P=.40)$ (Table). When all 3 procedural areas were combined, there was a significant 
TABLE Model-Adjusted Percentages of DNR Acknowledgment

\begin{tabular}{|c|c|c|c|c|c|c|}
\hline & \multicolumn{2}{|c|}{ Gastroenterology } & \multicolumn{2}{|c|}{ Cardiology } & \multicolumn{2}{|c|}{ Interventional Radiology } \\
\hline & Before & After & Before & After & Before & After \\
\hline Procedures, No. & 2,872 & 884 & 919 & 458 & 1,571 & 590 \\
\hline DNR patients, No. (\%) & $63(2.2)$ & $43(4.9)$ & $41(4.5)$ & $24(5.2)$ & $107(6.8)$ & $76(13)$ \\
\hline $\begin{array}{l}\text { DNR acknowledgment } \\
\text { (adjusted), \% }\end{array}$ & 46 & 53.5 & 75.6 & 91.7 & 7.5 & $26.3^{a}$ \\
\hline
\end{tabular}

improvement in the overall percentage of DNR acknowledgment postintervention from $38.6 \%$ to $61.1 . \%(P=.01)$.

\section{DISCUSSION}

With the LSTDI, DNR orders remain in place and are valid in the inpatient and outpatient setting until reversed by the patient. This creates new challenges for proceduralists. Before our intervention, only about one-third of proceduralists' recognized DNR status before procedures. This low rate of preprocedural DNR acknowledgments is not unique to the VA. A pilot study assessing rate of documentation of code status discussions in patients undergoing venting gastrostomy tube for malignant bowel obstruction showed documentation in only $22 \%$ of cases before the procedure. ${ }^{10}$ Another simulation-based study of anesthesiologist showed only $57 \%$ of subjects addressed resuscitation before starting the procedure. ${ }^{11}$

Despite the low initial rates of DNR acknowledgment, our intervention successfully improved these rates, although with variation between procedural areas. Prior studies looking at improving adherence to guidelines have shown the benefit of physician education. ${ }^{12,13}$ Improving code status acknowledgment before an invasive procedure not only involves increasing awareness of a preexisting code status, but also developing a system to incorporate the documentation process efficiently into the procedural workflow and ensuring that providers are aware of the appropriate process. Although the largest improvement was in interventional radiology, many patients postintervention still did not have their DNR orders acknowledged. Confusion is created when the patient is cared for by a different HCP or when the resuscitation team is called during a cardiac arrest. Cardio- pulmonary resuscitation may be started or withheld incorrectly if the patient's most recent wishes for resuscitation are unclear. ${ }^{14}$

Outside of using education to raise awareness, other improvements could utilize informatics solutions, such as developing an alert on opening a patient chart if a DNR status exists (such as a pop-up screen) or adding code status as an item to a preprocedural checklist. Similar to our study, previous studies also have found that a systematic approach with guidelines and templates improved rates of documentation of code status and DNR decisions. ${ }^{15,16} \mathrm{~A}$ large proportion of the LST notes and procedures done on patients with a DNR in our study occurred in the inpatient setting without any involvement of the primary care provider in the discussion. Having an automated way to alert the primary care provider that a new LST note has been completed may be helpful in guiding future care. Future work could identify additional systematic methods to increase acknowledgment of DNR.

\section{Limitations}

Our single-center results may not be generalizable. Although the interaction between procedural area and time was tested, it is possible that improvement in DNR acknowledgment was attributable to secular trends and not the intervention. Other limitations included the decreased generalizability of a VA health care initiative and its unique electronic health record, incomplete attendance rates at our educational sessions, and a lack of patient-centered outcomes.

\section{CONCLUSIONS}

A templated addendum combined with targeted staff education improved the percentage of DNR acknowledgments before 
nonsurgical invasive procedures, an important step in establishing patient preferences for life-sustaining treatment in procedures with potential complications. Further research is needed to assess whether these improvements also lead to improved patientcentered outcomes.

\section{Acknowledgments}

The authors would like to acknowledge the invaluable help of Dr. Kathryn Rice and Dr. Anne Melzer for their guidance in the manuscript revision process.

\section{Author disclosures}

The authors report no actual or potential conflicts of interest with regard to this article.

\section{Disclaimer}

The opinions expressed herein are those of the authors and do not necessarily reflect those of Federal Practitioner, Frontline Medical Communications Inc., the US Government, or any of its agencies.

\section{References}

1. Physician Orders for Life-Sustaining Treatment Paradigm. Honoring the wishes of those with serious illness and frailty. Accessed January 11, 2021.

2. Arepally A, Oechsle D, Kirkwood S, Savader S. Safety of conscious sedation in interventional radiology. Cardiovasc Intervent Radiol. 2001;24(3):185-190. doi:10.1007/s002700002549

3. Arrowsmith J, Gertsman B, Fleischer D, Benjamin S. Results from the American Society for Gastrointestinal Endoscopy/U.S. Food and Drug Administration collaborative study on complication rates and drug use during gastrointestinal endoscopy. Gastrointest Endosc. 1991;37(4):421-427. doi:10.1016/s0016-5107(91)70773-6

4. Burkle C, Swetz K, Armstrong M, Keegan M. Patient and doctor attitudes and beliefs concerning perioperative do not resuscitate orders: anesthesiologists' growing compliance with patient autonomy and selfdetermination guidelines. BMC Anesthesiol. 2013;13:2. doi:10.1186/1471-2253-13-2

5. American College of Surgeons. Statement on advance directives by patients: "do not resuscitate" in the operative room. Published January 3, 2014. Accessed January 11, 2021. https://bulletin.facs.org/2014/01/statement-on -advance-directives-by-patients-do-not-resuscitate-in-theoperating-room
6. Association of periOperative Registered Nurses. AORN position statement on perioperative care of patients with do-not-resuscitate or allow-natural death orders. Reaffirmed February 2020. Accessed June 16, 2020. https://www.aorn.org/guidelines/clinical-resources /position-statements

7. Bastron DR. Ethical guidelines for the anesthesia care of patients with do-not-resuscitate orders or other directives that limit treatment. Published 1996. Accessed January 11, 2021. https://pubs.asahq.org/anesthesiology /article/85/5/1190/35862/Ethical-Concerns-in-Anesthetic -Care-for-Patients

8. Baxter L, Hancox J, King B, Powell A, Tolley T. Stop! Patients receiving CPR despite valid DNACPR documentation. Eur J Pall Car. 2018;23(3):125-127.

9. Agency for Healthcare Research and Quality. Practice facilitation handbook, module 10: academic detailing as a quality improvement tool. Last reviewed May 2013. Accessed January 11, 2021. 2021. https://www.ahrq.gov /ncepcr/tools/pf-handbook/mod10.html

10. Urman R, Lilley E, Changala M, Lindvall C, Hepner D, Bader A. A pilot study to evaluate compliance with guidelines for preprocedural reconsideration of code status limitations. J Palliat Med. 2018;21(8):1152-1156. doi:10.1089/jpm.2017.0601

11. Waisel D, Simon R, Truog R, Baboolal H, Raemer D. Anesthesiologist management of perioperative do-not-resuscitate orders: a simulation-based experiment. Simul Healthc. 2009;4(2):70-76. doi:10.1097/SIH.0b013e31819e137b

12. Lozano P, Finkelstein J, Carey V, et al. A multisite randomized trial of the effects of physician education and organizational change in chronic-asthma care. Arch Pediatr Adolesc Med. 2004;158(9):875-883. doi:10.1001/archpedi.158.9.875

13. Brunström M, Ng N, Dahlström J, et al. Association of physician education and feedback on hypertension management with patient blood pressure and hypertension control. JAMA Netw Open. 2020;3(1):e1918625. doi:10.1001/jamanetworkopen.2019.18625

14. Wong J, Duane P, Ingraham N. A case series of patients who were do not resuscitate but underwent cardiopulmonary resuscitation. Resuscitation. 2020;146:145-146. doi:10.1016/j.resuscitation.2019.11.020

15. Mittelberger J, Lo B, Martin D, Uhlmann R. Impact of a procedure-specific do not resuscitate order form on documentation of do not resuscitate orders. Arch Intern Med. 1993;153(2):228-232.

16. Neubauer M, Taniguchi $\mathrm{C}$, Hoverman J. Improving incidence of code status documentation through process and discipline. J Oncol Pract. 2015;11(2):e263-266. doi:10.1200/JOP.2014.001438 